\title{
Multiferroics march on
}

\author{
Much academic and industrial effort has been devoted to the study of multiferroics, but if related technologies are \\ to have real-world impact, market awareness and reproducibility are also key.
}

W e are all familiar with magnets, materials that possess spontaneous magnetization due to the presence of magnetic dipoles. If these dipoles all align in the same direction this is ferromagnetic ordering, and the direction of the dipoles can be changed by applying an external magnetic field. Similar behaviour can also be observed with ferroelectrics, which possess spontaneous electric polarization that can be reversed by electric field, or ferroelastics, which possess spontaneous strain that can be reversed by stress.

These are all examples of ferroics, which show spontaneous order and a historydependent switching behaviour; and ferroic orders are by no means exclusive. Coupling electric and magnetic fields in a material was first proposed in the nineteenth century ${ }^{1}$ and the first experimental attempts to dope magnetic ions into a ferroelectric occurred in $1958^{2}$. Multiferroics combine more than one ferroic order, and this can induce new properties. Of particular interest for electronic devices are magnetoelectric multiferroics, where applied electric fields change magnetic properties and vice versa. However, these are rare as the mechanisms for ferroelectricity and magnetism tend to oppose each other ${ }^{3}$. In this issue, a series of pieces explore the properties and applications of multiferroics.

In a Review, Nicola Spaldin and Ramamoorthy Ramesh discuss the different possible routes to generating magnetoelectric multiferroicity in materials. Ferroelectricity is generated by distortions that break centrosymmetry - for example, ionic repulsion driven by the stereoactive lone pair of electrons, or empty $d$-orbitals that favour off-centring by energy-lowering hybridization with the surrounding anion lattice - while magnetism requires partially filled $d$ - or $f$-orbitals to allow ordering of localized electronic states. Beyond the fundamental interest in understanding and mastering multiferroic properties, how research is getting closer to impacting practical applications such as logic-memory devices is also reviewed. Switching magnetic state by applied electric rather than magnetic field in these devices requires much less energy ${ }^{4}$, but a limited number of compounds present technologically useful room-temperature multiferroic behaviour. A key requirement to make magnetoelectric logic memory competitive to alternative technologies would be switching of

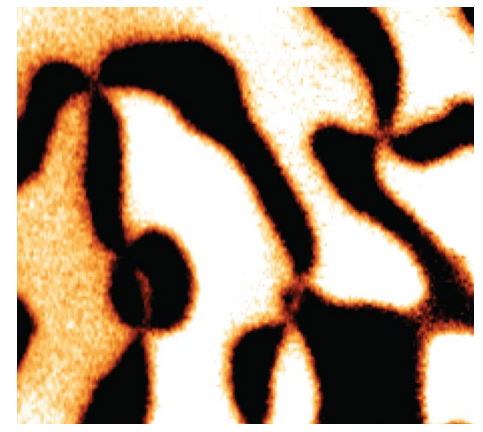

Direct visualization of magnetoelectric domains under magnetic field in multiferroic $h-\mathrm{ErMnO}_{3}$. Adapted from ref. ${ }^{10}$, Springer Nature Ltd.

ferroelectric properties with small $(100 \mathrm{mV})$ voltages; a greater choice of magnetoelectric materials would be useful to mature devices and could help develop new device concepts.

An approach to develop new functionality, and thus devices, is to design and manipulate topological structures, such as the domain walls that separate different ferroelectric domains, or the skyrmions that form in magnetic materials. These structures can not only pre-exist in a material but also be engineered, and so used to carry information or act as electronically conducting elements in novel device concepts. In a Comment, Jan Seidel discusses the formation, engineering and application of topological structures in ferroics for electronics applications.

Although multiferroicity can exist in a single material, it can also be present in composites formed by combining ferroelectric and magnetic materials. These are useful for applications such as sensors or antennae. In an interview, Nian Sun discusses materials requirements for these applications, and notes that targeting specific magnetoelectric properties in thin films is even more challenging than fabrication processes for other semiconductor-based devices. But as important as the development of a technology are the issues involved with pushing that technology to the market, efforts must also be made to educate the end users about the benefits of a technology.

In some cases the technology may rely on fundamental principles far outside everyday experience. For example, some ferroelectrics or magnets display quantum critical behaviour, where a phase transition can occur at zero kelvin, but even so the quantum critical region can impact materials properties at high temperature ${ }^{5}$. An Article by Nicola Spaldin and colleagues introduces the phenomenology of quantum criticality in multiferroics, and predicts candidate perovskite materials where this can occur. In her associated News \& Views, Premala Chandra observes that a multiferroic quantum critical point could result in a large response from an imposed electric field when the material is cooled, of potential interest for electrocaloric applications.

Research in multiferroics has continued growing apace since $2007^{6}$. There have been advances in fundamental understanding, such as the observation of a fourth form of ferroic order, ferrotoroidicity, the spontaneous ordering of magnetic vortices ${ }^{7}$, and the finding that domain walls can have electrical conductivity different from the bulk, relevant for memory applications ${ }^{8}$. There have also been developments in processing, with advances in film-deposition techniques, enabling the controlled layer-by-layer growth of composite materials that can possess tailored properties, such as room-temperature electric-field control of magnetism ${ }^{9}$.

There is no doubt that the study of multiferroics has resulted in the acquisition of considerable scientific knowledge from basic research. However, there has been a relative paucity in the broad uptake and commercialization of multiferroic and the pace of commercial innovation in this field must be accelerated. For multiferroics to have an impact outside the academic world, applications must be further developed. We hope this overview triggers further materials discovery for developing applications, as well as further understanding of these systems through fundamental research.

Published online: 19 February 2019 https://doi.org/10.1038/s41563-019-0310-y

References

1. Curie, P. J. Phys. Theor. Appl. 3, 393-415 (1894).

2. Smolenskii, G. A., Isupov, V. A., Krainik, N. N. \& Agranovskaya, A. I. Izv. Akad. Nauk SSSR 25, 1333-1339 (1961).

3. Hill, N. A. J. Phys. Chem. B 104, 6694-6709 (2000).

4. Manipatruni, S., Nikonov, D. E. \& Young, I. A. Nat. Phys. 14 338-343 (2018).

5. Risschau, C. W. Nat. Phys. 13, 643-648 (2017).

6. Multiferroics. Nat. Mater. https://www.nature.com/collections/ pqghwqwqzp (2007)

7. Van Aken, B. B., Rivera, J.-P., Schmid, H. \& Fiebig, M. Nature 449, 702-705 (2007).

8. Jiang, J. et al. Nat. Mater. 17, 49-56 (2018).

. Mundy, J. A. et al. Nature 537, 523-527 (2016)

10. Geng, Y. et al. Nat. Mater. 13, 163-167 (2014). 